\title{
FINAL EVOLUTIONS FOR SIMPLIFIED MULTISTRAIN/TWO-STREAM MODEL FOR TUBERCULOSIS AND DENGUE FEVER
}

\author{
JAUME LLIBRE, REGILENE D.S. OLIVEIRA, AND CLAUDIA VALLS
}

\begin{abstract}
The simplified multistrain/two-stream model for the tuberculosis and the Dengue fever here considered has three compartments, one susceptible and the other two infectious. We characterize all the final evolutions of this model under generic assumptions.
\end{abstract}

\section{Introduction AND STATEMENT OF the MAin RESUlts}

Recently it has become important the study of the resistant viral and bacterial strains, and the treatment on their proliferation, see for instance $[1,2]$. A way for analyzing these systems is through the multistrain model, which is a simplification of the model considered by CastilloChavez and Feng, see section 12 of [2], for the study of the tuberculosis model of Feng-VelascoHernández [5] for analyzing the Dengue fever. Driessche and Watmough [4] studied this model in terms of their reprodutive numbers and subthreshold epidemic equilibrium points.

This simplified model has a unique susceptible compartment (S), but has two infectious compartiment agents $\left(I_{1}, I_{2}\right)$. The equation of this simplified model is

$$
\begin{aligned}
\dot{I}_{1} & =\beta_{1} I_{1} S-\left(b+\gamma_{1}\right) I_{1}+\nu I_{1} I_{2}, \\
\dot{I}_{2} & =\beta_{2} I_{2} S-\left(b+\gamma_{2}\right) I_{2}-\nu I_{1} I_{2}, \\
\dot{S} & =b-b S+\gamma_{1} I_{1}+\gamma_{2} I_{2}-\left(\beta_{1} I_{1}+\beta_{2} I_{2}\right) S .
\end{aligned}
$$

From their biological meaning the parameters of this system satisfy

$$
\nu, b>0, \quad \beta_{i} \geq 0 \quad \text { and } \quad b+\gamma_{i}>0 \quad \text { for } i=1,2 .
$$

System (1) has the Darboux invariant

$$
I=\left(I_{1}+I_{2}+S-1\right) e^{b t} .
$$

See section 2 for a definition of Darboux invariant. Let $Q^{*}$ be the open octant $\left\{I_{1}>0\right\} \cap\left\{I_{2}>0\right\}$ in the space $\mathbb{R}^{3}$ of coordinates $\left(I_{1}, I_{2}, S\right)$. We shall see that the existence of this invariant implies that all the final evolutions of $I_{1}(t)$ and $I_{2}(t)$ when $t \rightarrow+\infty$ tend to the attractors contained in the quadrant $Q=\left\{I_{1}+I_{2}+S=1\right\} \cap\left\{I_{1} \geq 0\right\} \cap\left\{I_{2} \geq 0\right\}$ adding the infinity, see the definition of final evolution in section 2 .

We recall that an equilibrium point $p$ of a differential system is hyperbolic if the real part of the eigenvalues of the linear part of that system at $p$ are non-zero. As we shall see in section 3 the assumption that the equilibrium points of system (1) are hyperbolic is generic.

Key words and phrases. Mathematical epidemiology, tuberculosis, Dengue fever, final evolutions. 
In order to state our results on the final evolutions of system (1) we need to introduce some definitions and some conditions. Let

$$
\begin{aligned}
& I_{0}=\beta_{1}-\beta_{2}-\nu, \\
& I_{1}=\beta_{1}-b-\gamma_{1}, \\
& I_{2}=\beta_{2}-b-\gamma_{2}, \\
& I_{3}=\beta_{2}\left(b+\gamma_{1}-\nu\right)+\left(b+\gamma_{2}\right)\left(\nu-\beta_{1}\right), \\
& I_{4}=\beta_{1}\left(b+\gamma_{2}+\nu\right)-\left(b+\gamma_{1}\right)\left(\nu+\beta_{2}\right)
\end{aligned}
$$

and consider the following conditions

(I) $I_{0}>0, \quad I_{1}>0, \quad I_{2}>0, \quad I_{3}>0, \quad I_{4}>0$,

(II) $I_{0} \neq 0, \quad I_{1}>0, \quad I_{2}>0, \quad I_{3}<0, \quad I_{4}>0$,

(III) $I_{0} \neq 0, \quad I_{1}>0, \quad I_{2}>0, \quad I_{3}>0, \quad I_{4}<0$,

(IV) $I_{0} \neq 0, \quad I_{1}<0, \quad I_{2}<0, \quad$ and $I_{3}<0 \quad$ or $\quad I_{4}<0$.

Under any of the conditions (I)-(IV), system (1) has the following four equilibrium points

$$
\begin{aligned}
& p_{0}=(0,0,1), \\
& p_{1}=\left(\frac{I_{1}}{\beta_{1}}, 0, \frac{b+\gamma_{1}}{\beta_{1}}\right), \\
& p_{2}=\left(0, \frac{I_{2}}{\beta_{2}}, \frac{b+\gamma_{2}}{\beta_{2}}\right), \\
& p_{3}=\left(\frac{I_{3}}{\nu I_{0}}, \frac{I_{4}}{\nu I_{0}}, \frac{\gamma_{1}-\gamma_{2}-1}{I_{0}}\right) .
\end{aligned}
$$

Our main result is the following.

Theorem 1. Assume that all equilibrium points of system (1) satisfying conditions (2) in the closure of the quadrant $Q$ adding the infinity, are hyperbolic. Then the following statements hold.

(a) Under assumptions $(I)$, except for $p_{3}$ or for the initial conditions which are on the stable manifold of $p_{3}$, all other initial conditions in $Q^{*}$ satisfy that $\left(I_{1}(t), I_{2}(t), S(t)\right)$ tends to the equilibria $p_{1}$ or $p_{2}$ when $t \rightarrow+\infty$. The bassins of attraction of $p_{1}$ and $p_{2}$ in $Q^{*}$ are separated by the stable manifolds of $p_{3}$.

(b) Under assumptions (II), for all initial conditions in $Q^{*}$ we have $\left(I_{1}(t), I_{2}(t), S(t)\right) \rightarrow p_{1}$ when $t \rightarrow+\infty$.

(c) Under assumptions (III), for all initial conditions in $Q^{*}$ we have $\left.\left(I_{1}(t), I_{2}(t), S(t)\right)\right)$ $\rightarrow p_{2}$ when $t \rightarrow+\infty$.

(d) Under assumptions $(I V)$, for all initial conditions in $Q^{*}$ we have $\left.\left(I_{1}(t), I_{2}(t), S(t)\right)\right) \rightarrow p_{0}$ when $t \rightarrow+\infty$. 
(e) For system (1) satisfying conditions (2) only the assumptions of statements $(a),(b),(c)$ and $(d)$ hold.

Theorem 1 is proved in section 3 .

We remark that the statement of Theorem 1 under our generic assumptions yields that, all the equilibrium points of the quadrant $Q$ adding the infinity are hyperbolic. It also provides the final evolution for the infectious agents $I_{1}$ and $I_{2}$ according to the different initial conditions.

\section{BASIC DEFINITIONS AND RESUlTS}

2.1. Invariant surface. We say that $f\left(I_{1}, I_{2}, S\right)=0$ is an invariant surface of system (1) if

$$
\frac{\partial f}{\partial I_{1}} \dot{I}_{1}+\frac{\partial f}{\partial I_{2}} \dot{I}_{2}+\frac{\partial f}{\partial S} \dot{S}=K\left(I_{1}, I_{2}, S\right) f
$$

where $K\left(I_{1}, I_{2}, S\right)$ is a polynomial. We note that an invariant surface has the property that if a trajectory of system (1) has a point on this surface the whole trajectory is contained in the surface.

Lemma 2. The plane $I_{1}+I_{2}+S-1=0$ is an invariant surface for system (1).

Proof. The proof follows easily from the definition of invariant surface, where $K\left(I_{1}, I_{2}, S\right)$ is the constant $-b$.

We note that the existence of this invariant plane is due to Nucci and Leach [7].

2.2. Darboux invariant. A function $I\left(I_{1}, I_{2}, S, t\right)$ is an invariant of system (1) if it is constant on the solutions of the system, i.e.

$$
\frac{d I\left(I_{1}, I_{2}, S, t\right)}{d t}=\frac{\partial I}{\partial I_{1}} \dot{I}_{1}+\frac{\partial I}{\partial I_{2}} \dot{I}_{2}+\frac{\partial I}{\partial S} \dot{S}+\frac{\partial I}{\partial t}=0
$$

on the trajectories of the system. When $I\left(I_{1}, I_{2}, S, t\right)=g\left(I_{1}, I_{2}, S\right) e^{k t}$ where $k$ is a non-zero real constant, we say that the invariant $I$ is a Darboux invariant.

Lemma 3. The function $I=\left(I_{1}+I_{2}+S-1\right) e^{b t}$ is a Darboux invariant for system (1).

Proof. It follows immediately from the definition of Darboux invariant.

2.3. The dynamics on the invariant plane. Since the plane $I_{1}+I_{2}+S-1=0$ is invariant for system (1) we shall study the dynamics of this system on this plane. So the equations of system (1) on this invariant plane are

$$
\begin{aligned}
& \dot{I}_{1}=I_{1}\left(\beta_{1}-\sigma_{1}-\beta_{1} I_{1}+\left(\nu-\beta_{1}\right) I_{2}\right), \\
& \dot{I}_{2}=I_{2}\left(\beta_{2}-\sigma_{2}-\left(\nu+\beta_{2}\right) I_{1}-\beta_{2} I_{2}\right),
\end{aligned}
$$

where $\sigma_{i}=b+\gamma_{i}$ for $i=1,2$.

The quadrant $Q$ on the invariant plane $I_{1}+I_{2}+S-1=0$ is given by $\left\{\left(I_{1}, I_{2}\right): I_{1} \geq 0, I_{2} \geq 0\right\}$. To define the closure of $Q$ adding the infinity (see Section 2.5) we shall use the so called Poincaré 
compactification of a polynomial differential system. Note that system (3) is a polynomial differential system of degree 2 in the variables $I_{1}$ and $I_{2}$.

\subsection{The compactification of the quadrant $Q$. Let}

$$
\mathcal{X}=P(x, y) \frac{\partial}{\partial x}+Q(x, y) \frac{\partial}{\partial y}
$$

be the planar polynomial vector field of degree 2 associated to the polynomial differential system

$$
\dot{x}=P(x, y), \quad \dot{y}=Q(x, y) .
$$

The Poincaré compactified vector field $p(\mathcal{X})$ of $\mathcal{X}$ is an analytic vector field defined on the sphere $\mathbb{S}^{2}$ as follows. For more details on this compactification see the chapter 5 of the book [6].

Let $\mathbb{S}^{2}=\left\{y=\left(y_{1}, y_{2}, y_{3}\right) \in \mathbb{R}^{3} ; y_{1}^{2}+y_{2}^{2}+y_{3}^{2}=1\right\}$ and $T_{y} \mathbb{S}^{2}$ be the tangent plane to $\mathbb{S}^{2}$ at point $y$. The plane of definition of $\mathcal{X}$ is identified with the plane $T_{(0,0,1)} \mathbb{S}^{2}$ and let $f: T_{(0,0,1)} \mathbb{S}^{2} \rightarrow \mathbb{S}^{2}$ be the central projection. Using the map $f$ we get two copies of $\mathcal{X}$ on $\mathbb{S}^{2}$ one in the northern hemisphere and the other in the southern one. Let $\mathcal{X}^{\prime}$ be the vector field $D(f) \circ \mathcal{X}$ on $\mathbb{S}^{2} \backslash \mathbb{S}^{1}$. Here $\mathbb{S}^{1}=\left\{y \in \mathbb{S}^{2} ; y_{3}=0\right\}$ is the equator of $\mathbb{S}^{2}$ and corresponds to the infinity of the plane of definition of $\mathcal{X}$.

The vector field $p(\mathcal{X})$ is the unique analytical extension of $y_{3}^{2} \mathcal{X}^{\prime}$ to $\mathbb{S}^{2}$. On each hemisphere of $\mathbb{S}^{2} \backslash \mathbb{S}^{1}$ there is a copy of $\mathcal{X}$. The dynamics of $p(\mathcal{X})$ near $\mathbb{S}^{1}$ provides the dynamics of $\mathcal{X}$ near the infinity. The infinity $\mathbb{S}^{1}$ is invariant by the Poincaré compactification $p(\mathcal{X})$. The projection $\left(y_{1}, y_{2}, y_{3}\right) \mapsto\left(y_{1}, y_{2}\right)$ of the closed northern hemisphere on $y_{3}=0$ is the Poincaré disc. Note that its boundary is $\mathbb{S}^{1}$.

In order to have the equations of $p(\mathcal{X})$ on $\mathbb{S}^{2}$ we take six local charts $\left(U_{i}, F_{i}\right)$ and $\left(V_{i}, G_{i}\right)$, where $U_{i}=\left\{y \in \mathbb{S}^{2} ; y_{i}>0\right\}, V_{i}=\left\{y \in \mathbb{S}^{2} ; y_{i}<0\right\}$, the diffeomorphisms $F_{i}: U_{i} \rightarrow \mathbb{R}^{2}$ and $G_{i}: V_{i} \rightarrow \mathbb{R}^{2}$, for $i=1,2,3$, which are the inverses of the central projections from the tangent planes at the points $(1,0,0),(-1,0,0),(0,1,0),(0,-1,0),(0,0,1)$ and $(0,0,-1)$, respectively. If we denote the points of $U_{i}$ and $V_{i}$ by $z=(u, v)$, then $z$ has different meaning depending on the local charts that we are working. Therefore, after easy computations the differential systems associated to the vector field $p(\mathcal{X})$ are

$$
\begin{gathered}
v^{2} \Delta(z)\left(Q\left(\frac{1}{v}, \frac{u}{v}\right)-u P\left(\frac{1}{v}, \frac{u}{v}\right),-v P\left(\frac{1}{v}, \frac{u}{v}\right)\right) \text { in } U_{1}, \\
v^{2} \Delta(z)\left(P\left(\frac{u}{v}, \frac{1}{v}\right)-u Q\left(\frac{u}{v}, \frac{1}{v}\right),-v Q\left(\frac{u}{v}, \frac{1}{v}\right)\right) \text { in } U_{2}, \\
\Delta(z)(P(u, v), Q(u, v)) \text { in } U_{3},
\end{gathered}
$$

where $\Delta(z)=1 / \sqrt{u^{2}+v^{2}+1}$. For the $V_{i}$ 's local charts the expressions are the same as the ones of $U_{i}$ 's changed of sign.

Note that $v=0$ always denotes the points at the infinity $\mathbb{S}^{1}$ in the local charts $U_{1}, V_{1}, U_{2}$ and $V_{2}$. From now on we cancel the factor $\Delta(z)>0$ from the expressions of $p(\mathcal{X})$ in the local charts doing a rescaling of the time.

The equilibrium points of $p(\mathcal{X})$ which are in $\mathbb{S}^{1}$ are called infinite equilibrium points of $p(\mathcal{X})$ or of $\mathcal{X}$. 
2.5. The Poincaré compactification of system (3). The equations of system (3) on the invariant plane $I_{1}+I_{2}+S-1=0$ in the local charts $U_{1}$ and $U_{2}$ write, respecively, as

$$
\begin{aligned}
& \dot{u}=u\left(\left(\beta_{1}-\beta_{2}-\nu\right)(1+u)+\left(\sigma_{1}-\sigma_{2}-\beta_{1}+\beta_{2}\right) v\right), \\
& \dot{v}=v\left(\beta_{1}+\left(\beta_{1}-\nu\right) u+\left(\sigma_{1}-\beta_{1}\right) v\right),
\end{aligned}
$$

and

$$
\begin{aligned}
& \dot{u}=-u\left(\left(\beta_{1}-\beta_{2}-\nu\right)(1+u)+\left(\sigma_{1}-\sigma_{2}-\beta_{1}+\beta_{2}\right) v\right), \\
& \dot{v}=v\left(\beta_{2}+\left(\beta_{2}+\nu\right) u+\left(\sigma_{2}-\beta_{2}\right) v\right) .
\end{aligned}
$$

Since the straight lines $I_{1}=0$ and $I_{2}=0$ of system (3) are invariant and we are only interested in the dynamics when $I_{1} \geq 0$ and $I_{2} \geq 0$, we restrict our attention to the quadrant $Q$ which is open at infinity. Doing the Poincaré compactification of system (3) we shall work in the closure of $Q$ in the Poincaré disc, this closure is the one that we have mentioned in section 1 saying that we consider the closure of $Q$ adding the infinity. This closure in what follows will be denoted by $\bar{Q}$.

It is easy to see that system (5) (respectively (6)) has a unique infinite equilibrium point $u_{1}$ (respectively $u_{2}$ ) in $\bar{Q}$, namely the origin $(0,0)$ of the chart $U_{1}$ (respectively $U_{2}$ ).

The Poincaré compactification can also be done for the polynomial differential system (1) defined in $\mathbb{R}^{3}$, see for instance [3]. Then we shall denote the closure of $Q^{*}$ adding the infinity as $\overline{Q^{*}}$.

Let $\mathcal{X}_{3}$ and $\mathcal{X}_{2}$ be the polynomial vector fields associated to the polynomial differential systems (1) and (3), respectively. We note that the orbits of the compactified vector fields $p\left(\mathcal{X}_{3}\right)$ and $p\left(\mathcal{X}_{2}\right)$ on $\overline{Q^{*}}$ and $\bar{Q}$, respectively, are defined for $t \in(-\infty,+\infty)$, because $\overline{Q^{*}}$ and $\bar{Q}$ are compact sets. This a classical result which for instance follows easily from Theorem 1.2 of [6].

2.6. Final evolutions. Let $\phi_{p}(t)=\left(I_{1}(t), I_{2}(t), S(t)\right)$ be a solution of $p\left(\mathcal{X}_{3}\right)$. We define the $\omega$-limit and the $\alpha$-limit set of $p$, denoted by $\omega(p)$ and $\alpha(p)$ respectively, as

$$
\begin{aligned}
& \omega(p)=\left\{q \in \overline{Q^{*}}: \exists\left\{t_{n}\right\} \text { with } t_{n} \rightarrow+\infty \text { and } \phi_{p}\left(t_{n}\right) \rightarrow q \text { when } n \rightarrow+\infty\right\}, \\
& \alpha(p)=\left\{q \in \overline{Q^{*}}: \exists\left\{t_{n}\right\} \text { with } t_{n} \rightarrow-\infty \text { and } \phi_{p}\left(t_{n}\right) \rightarrow q \text { when } n \rightarrow+\infty\right\} .
\end{aligned}
$$

The $\omega$-limit of the solution $\phi_{p}(t)$ of $p\left(\mathcal{X}_{3}\right)$ with $p \in Q^{*}$ is called the final evolution of this solution.

The next proposition will show that all the final evolutions of the orbits starting at points of $Q^{*}$ are in $\bar{Q}$.

Proposition 4. Let $I\left(I_{1}, I_{2}, S, t\right)=\left(I_{1}+I_{2}+S-1\right) e^{b t}$ be a Darboux invariant of system (1). If $\phi_{p}(t)$ is the solution of $p\left(\mathcal{X}_{3}\right)$ with $p \in Q^{*}$, then $\omega(p) \in \bar{Q}$.

Proof. Set $\phi_{p}(t)=\left(I_{1}(t), I_{2}(t), S(t)\right)$. Since $I$ is a Darboux invariant $I\left(\phi_{p}(t)\right)$ is constant. But since $b>0$ and $e^{b t} \rightarrow+\infty$ when $t \rightarrow+\infty$, we have that $I_{1}(t)+I_{2}(t)+S(t)-1 \rightarrow 0$ when $t \rightarrow+\infty$. So $\omega(p) \in \bar{Q}$. 
Remark 5. Proposition 4 implies that in order to study the final evolutions of the orbits $\phi_{p}(t)$ of $p\left(\mathcal{X}_{3}\right)$ with $p \in Q^{*}$, it is sufficient to study the final evolutions of the orbits $\phi_{p}(t)$ of $p\left(\mathcal{X}_{2}\right)$ with $p \in Q$.

\section{Proof of Theorem 1}

The eigenvalues of the infinite and finite equilibrium points of the vector field $p\left(\mathcal{X}_{2}\right)$ contained in $\bar{Q}$ are: $I_{0}$ and $\beta_{1}$ for $u_{1},-I_{0}$ and $\beta_{2}$ for $u_{2}, I_{1}$ and $I_{2}$ for $p_{0},-I_{1}$ and $-I_{4} / \beta_{1}$ for $p_{1},-I_{2}$ and $-I_{3} / \beta_{2}$ for $p_{2}$, the roots of the polynomial $\lambda^{2}-T \lambda+D$, where

$$
T=-\frac{\beta_{1} I_{3}+\beta_{2} I_{4}}{\nu I_{0}}, \quad D=-\frac{I_{3} I_{4}}{\nu I_{0}},
$$

for $p_{3}$.

Since by assumptions our equilibrium points are hyperbolic, and from (2) we have that $I_{k} \neq 0$, for $k=0,1,2,3,4$, we conclude that $\beta_{1}, \beta_{2}>0$ and $\nu>0$.

By remark 5 we can reduce the proofs of the statements of Theorem 1 to study the phase portrait of $p\left(\mathcal{X}_{2}\right)$ in the quadrant $\bar{Q}$.

Proof of statement (a) of Theorem 1. Under assumption (I) and since the equilibrium points are hyperbolic by Theorem 2.15 of [6] we have that $u_{1}$ is an unstable node, $u_{2}$ is a saddle, $p_{0}$ is an unstable node, $p_{1}$ and $p_{2}$ are stable nodes, and since the determinant of the equilibrium point $p_{3}$ is negative, $p_{3}$ restricted to the invariant plane is a saddle. Then, by using the Poincaré Bendixson Theorem (see for instance Theorem 1.25 and Corollary 1.30 of [6]), the phase portrait of $p\left(\mathcal{X}_{2}\right)$ in $\bar{Q}$ is the one described in Figure 1 .

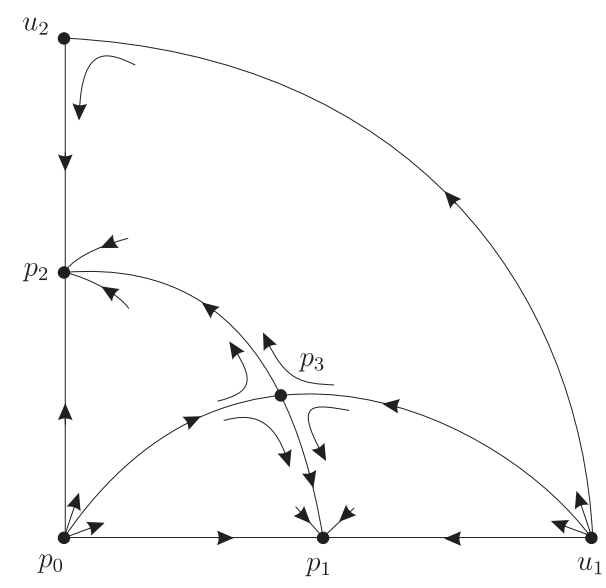

FiguRE 1. Phase portrait on the quadrant $\bar{Q}$ under the assumption (I).

In order to complete the proof of this statement we must compute the dimension of the invariant manifolds of the equilibrium point $p_{3}$ in $\overline{Q^{*}}$, which are the two eigenvalues of the saddle $p_{3}$ restricted to the invariant plane plus $-b$ outside this plane. Hence $p_{3}$ has two negative eigenvalues and one positive eigenvalue. Consequently the stable manifold of $p_{3}$ has 
dimension 2 and separate the bassins of attractions of the stable nodes $p_{1}$ and $p_{2}$ in $\overline{Q^{*}}$. By Remark 5 the proof of statement $(a)$ is done.

Proof of statement (b) of Theorem 1. Under assumption (II), $I_{0}>0$ (respectively $I_{0}<0$ ) and since the equilibrium points are hyperbolic we have that $u_{1}$ is an unstable node (respectively a saddle), $u_{2}$ is a saddle (respectively an unstable node), $p_{0}$ is an unstable node, $p_{1}$ is a stable node, and $p_{2}$ is saddle. Note that $p_{3}$ does not belong to $\bar{Q}$. Then, by the Poincaré-Bendixson Theorem the phase portrait of $p\left(\mathcal{X}_{2}\right)$ in $\bar{Q}$ is the one described in Figure 2(a) (respectively 2(b)). This completes the proof of statement $(b)$.

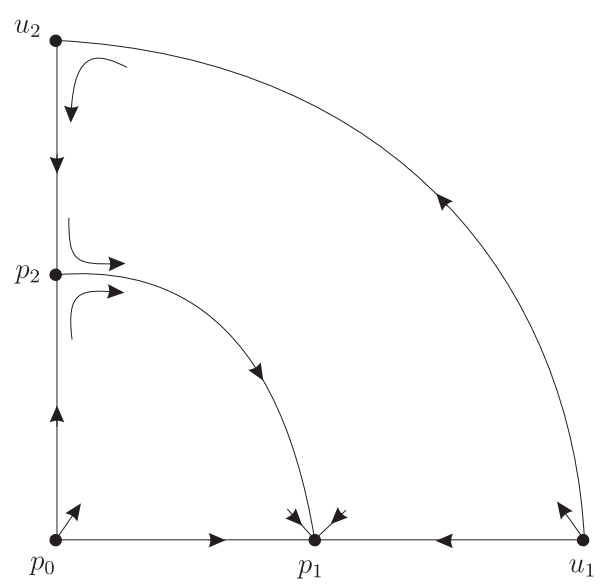

(a)

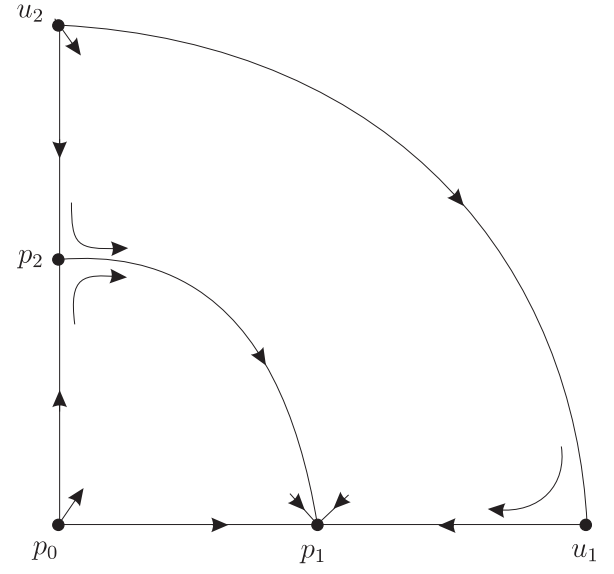

(b)

FIgURE 2. Phase portraits on the quadrant $\bar{Q}$ under the assumption (II) for $I_{0}>0$ in $(a)$ and for $I_{0}<0$ in $(b)$.

Proof of statement (c) of Theorem 1. Under assumption (III), $I_{0}>0$ (respectively $I_{0}<0$ ) and since the equilibrium points are hyperbolic we have that $u_{1}$ is an unstable node (respectively a saddle), $u_{2}$ is a saddle (respectively an unstable node), $p_{0}$ is an unstable node, $p_{1}$ is a saddle, and $p_{2}$ is stable node. Again $p_{3}$ does not belong to $\bar{Q}$. Then, by the Poincaré-Bendixson Theorem the phase portrait of $p\left(\mathcal{X}_{2}\right)$ in $\bar{Q}$ is the one described in Figure 3(a) (respectively $3(\mathrm{~b})$ ). This completes the proof of statement $(c)$.

Proof of statement $(d)$ of Theorem 1. Under assumption (IV), $I_{0}>0$ (respectively $I_{0}<0$ ) and since the equilibrium points are hyperbolic we have that $u_{1}$ is an unstable node (respectively a saddle), $u_{2}$ is a saddle (respectively an unstable node), and $p_{0}$ is a stable node. Now $p_{1}, p_{2}$ and $p_{3}$ do not belong to $\bar{Q}$. Then, by the Poincaré-Bendixson Theorem the phase portrait of $p\left(\mathcal{X}_{2}\right)$ in $\bar{Q}$ is the one described in Figure 4(a) (respectively 4(b)). This completes the proof of statement $(d)$.

Proof of statement (e) of Theorem 1. According to the signs of $I_{k}$ for $k=0,1,2,3,4$ the cases which are not considered in the statements $(a),(b),(c)$ and $(d)$ of Theorem (1) are the following 


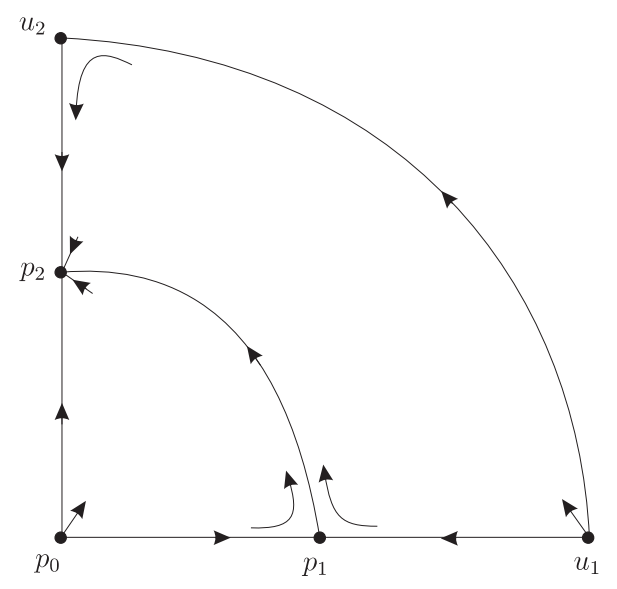

(a)

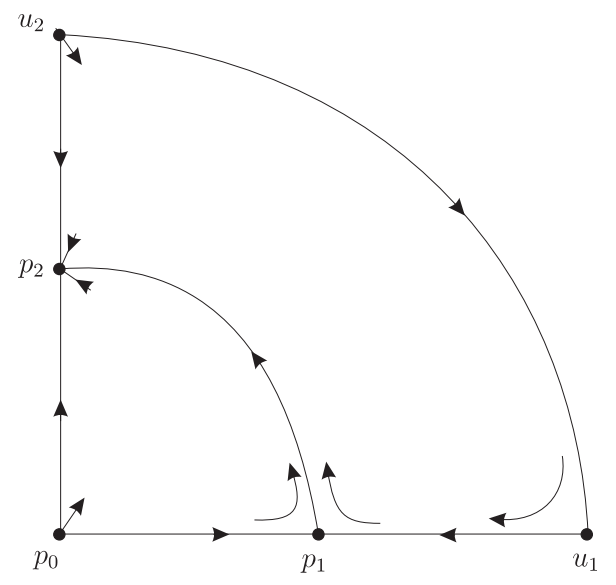

(b)

Figure 3. Phase portraits on the quadrant $\bar{Q}$ under the assumption (III) for $I_{0}>0$ in $(a)$ and for $I_{0}<0$ in $(b)$.

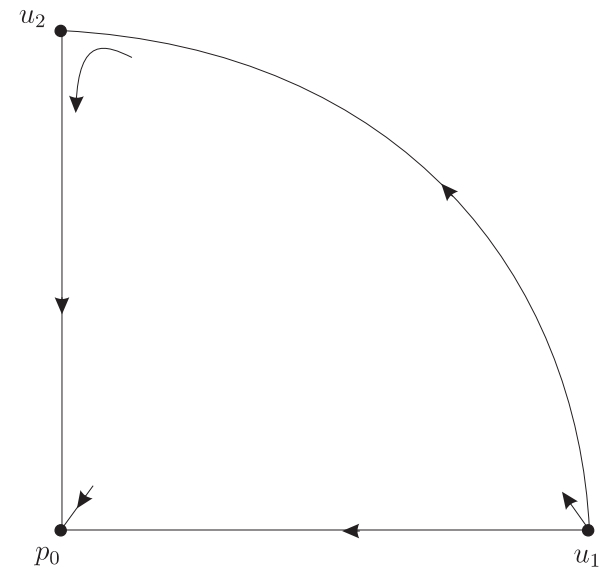

(a)

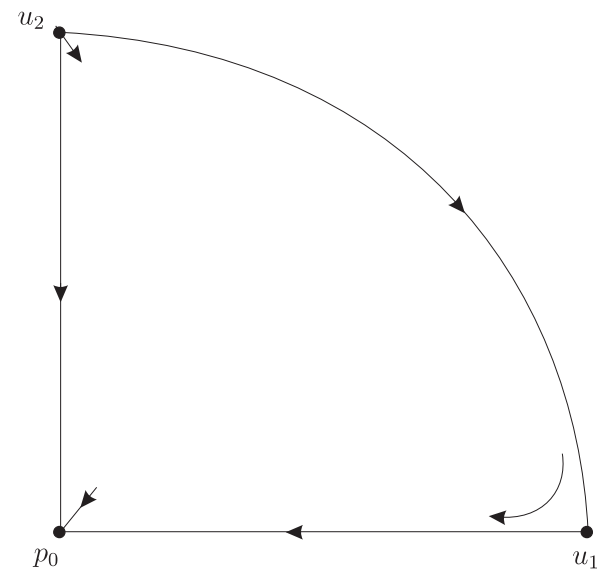

(b)

Figure 4. Phase portraits on the quadrant $\bar{Q}$ under the assumption (IV) for $I_{0}>0$ in (a) and for $I_{0}<0$ in $(b)$.

(V) $I_{0} \neq 0, \quad I_{1}<0, \quad I_{2}>0, \quad I_{3}>0, \quad I_{4}>0$,

(VI) $I_{0} \neq 0, \quad I_{1}>0, \quad I_{2}<0, \quad I_{3}>0, \quad I_{4}>0$,

(VII) $I_{0} \neq 0, \quad I_{1}<0, \quad I_{2}<0, \quad I_{3}>0, \quad I_{4}>0$,

(VIII) $I_{0}<0, \quad I_{1}>0, \quad I_{2}>0, \quad I_{3}>0, \quad I_{4}>0$.

Now we shall prove that these four cases are not realizable for system (3) satisfying (2), and this will complete the proof of statement $(e)$.

Under assumption $(\mathrm{V})$ with $I_{0}>0$ we have that $u_{1}$ is an unstable node, $u_{2}$ is a saddle, $p_{0}$ is saddle, $p_{2}$ is a stable node and $p_{3}$ is a saddle. In this case the point $p_{1}$ does not belong to 
$\bar{Q}$. Then by using the Poincaré-Bendixson Theorem we get a contradiction because the two unstable separatrices of the saddle $p_{3}$ go to $p_{2}$, and then the stable separatrix contained in the region limited by the two unstable separatrices cannot have its $\alpha$-limit.

Under assumption $(\mathrm{V})$ with $I_{0}<0$ we have that $u_{1}$ is a saddle, $u_{2}$ is an unstable node, $p_{0}$ is saddle, $p_{2}$ is a stable node and $p_{3}$ is a focus or a node. In this case the point $p_{1}$ does not belong to $\bar{Q}$. Then from the unstable node $u_{2}$ there is an orbit $\Gamma_{1}$ going to $u_{1}$ and an orbit $\Gamma_{2}$ going to $p_{0}$, the orbits starting at $u_{1}$ between $\Gamma_{1}$ and $\Gamma_{2}$ need a separatrice contained in the interior of $\bar{Q}$ due to the fact that $\Gamma_{1}$ and $\Gamma_{2}$ have different $\omega$-limits. But there is no separatrices in this case in the interior of $\bar{Q}$, and this is a contradiction.

The proof that the vector field $p\left(\mathcal{X}_{2}\right)$ cannot satisfy the assumption (VI) follows in the same way than the proof that under the assumptions $(\mathrm{V})$ the vector field $p\left(\mathcal{X}_{2}\right)$ cannot satisfy assumptions (V) but using the point $p_{1}$ of assumption $(V I)$ instead of the point $p_{2}$ of assumption $(\mathrm{V})$.

The proof that the vector field $p\left(\mathcal{X}_{2}\right)$ cannot satisfy the assumption (VII) with $I_{0}>0$ is the same than the proof under the assumption $(\mathrm{V})$ with $I_{0}>0$ but using the point $p_{2}$ instead of the point $p_{0}$. The proof that the vector field $p\left(\mathcal{X}_{2}\right)$ cannot satisfy the assumption (VII) with $I_{0}<0$ is the same than the proof under the assumption $(\mathrm{V})$ with $I_{0}<0$.

The proof that the vector field $p\left(\mathcal{X}_{2}\right)$ cannot satisfy the assumption (VIII) follows as the proof under the assumptions $(\mathrm{V})$ with $I_{0}<0$ using the point $p_{2}$ for the assumption (VIII) instead of the point $p_{0}$ for the assumption $(\mathrm{V})$.

This completes the proof of statement $(e)$ of Theorem 1.

3.1. Generic conditions. Under the assumptions that the finite and infinite equilibrium points in $\bar{Q}$ are hyperbolic we get that $I_{k}$ for $k=0,1,2,3,4$ cannot be zero. Then in the space of parameters

$$
\left(\beta_{1}, \beta_{2}, b+\gamma_{1}, b+\gamma_{2}, \nu\right) \in E:=[0,+\infty)^{2} \times(0,+\infty)^{3},
$$

the subspaces satisfying conditions (I), (II), (III) and (IV) are open sets, and the union of these subspaces is dense in $E$. This density is due to the fact that all the other possible conditions, which are conditions (V), (VI), (VII) and (VIII) are empty as we have proved in the proof of statement $(e)$ of Theorem 1. This shows that conditions (I), (II), (III) and (IV) are generic.

\section{Discussion}

We have studied the simplified multistrain/two-stream model for the tuberculosis and the Dengue fever with three compartiments, one susceptible and the other two infectious provided by the differential system (1). Under generic assumptions we have characterized in Theorem 1 all the final evolutions of this model. As we have proved there are only two possible final evolutions. In the first one only one of the two infectious compartiments tends to zero, and in the second one both infectious compartiments tend to zero. Additionally we characterize the conditions in function of the parameters of the system which provide these different final evolutions. 


\section{ACKNOWLEDGEMENTS}

The first author is partially supported by FEDER-MINECO grants MTM2016-77278-P and MTM2013-40998-P,and an AGAUR grant number 2014SGR-568. The first two authors are partially supported by CAPES grant number 88881.030454/2013-01 from the program CSF-PVE. The second author is partially supported by FAPESP grant "Projeto Temático" 2014/00304-2. The third author is partially supported by FCT/Portugal through UID/MAT/04459/2013.

\section{REFERENCES}

[1] S.M. Blower, P.M. Small And P.C. Hopewell, Control strategies for tuberculosis epidemics: new models for old problems, Science 273 (1996), 497-500.

[2] C.Castillo-Chavez And Z. Feng, To treat of not to treat: the case of tuberculosis, J. Math. Biol. 35 (1997), 629-656.

[3] A. Cima And J. Llibre, Bounded polynomial systems, Trans. Amer. Math. Soc. 318, (1990), 420-448.

[4] P. VAn den Driessche And James Watmough, Reproduction numbers and sub-threshold endemic equilibria for compartmental models of disease transmission, Math. Biosci. 180 (2002), 29-48.

[5] Z. Feng And J.X. Velasco-Hernández, Competitive exclusion in a vectorâhost model for the Dengue fever, J. Math. Biol.35 (1997), 523-544.

[6] F. Dumortier, J. Llibre And J. C. Artés, Qualitative theory of planar differential systems, Universitext, Springer, New York, 2006.

[7] M.C. NucCi AND P.G.L. Leach, Lie integrable cases of the simplified multistrain/two-stream model for tuberculosis and dengue fever, J. Math. Anal. Appl. 333 (2007), 430-449.

Departament de Matemàtiques, Universitat Autònoma de Barcelona, 08193 Bellaterra, Barcelona, Catalonia, Spain

E-mail address: jllibre@mat.uab.cat

Departamento de Matemática, Instituto de Ciências Matemáticas e Computação, Universidade de São Paulo, Avenida Trabalhador São Carlense, 400, 13566-590, São Carlos, SP, Brazil.

E-mail address: regilene@icmc.usp.br

Departamento de Matemática, Instituto Superior Técnico, Universidade de Lisboa, 1049-001 Lisboa, Portugal

E-mail address: cvalls@math.tecnico.ulisboa.pt 